\title{
Metagenomic and metatranscriptomic analysis of human prostate microbiota from patients with prostate cancer
}

\author{
Ye Feng ${ }^{1,2}$ (D) Varune Rohan Ramnarine ${ }^{3}$, Robert Bell ${ }^{3}$, Stanislav Volik ${ }^{3}$, Elai Davicioni ${ }^{4}$, Vanessa M. Hayes ${ }^{5,6,7}$,
} Shancheng Ren ${ }^{{ }^{*}}$ and Colin C. Collins ${ }^{3^{*}}$

\begin{abstract}
Background: Prostate cancer (PCa) is the most common malignant neoplasm among men in many countries. Since most precancerous and cancerous tissues show signs of inflammation, chronic bacterial prostatitis has been hypothesized to be a possible etiology. However, establishing a causal relationship between microbial inflammation and PCa requires a comprehensive analysis of the prostate microbiome. The aim of this study was to characterize the microbiome in prostate tissue of PCa patients and investigate its association with tumour clinical characteristics as well as host expression profiles.
\end{abstract}

Results: The metagenome and metatranscriptome of tumour and the adjacent benign tissues were assessed in 65 Chinese radical prostatectomy specimens. Escherichia, Propionibacterium, Acinetobacter and Pseudomonas were abundant in both metagenome and metatranscriptome, thus constituting the core of the prostate microbiome. The biodiversity of the microbiomes could not be differentiated between the matched tumour/benign specimens or between the tumour specimens of low and high Gleason Scores. The expression profile of ten Pseudomonas genes was strongly correlated with that of eight host small RNA genes; three of the RNA genes may negatively associate with metastasis. Few viruses could be identified from the prostate microbiomes.

Conclusions: This is the first study of the human prostate microbiome employing an integrated metagenomics and metatranscriptomics approach. In this Chinese cohort, both metagenome and metatranscriptome analyses showed a non-sterile microenvironment in the prostate of PCa patients, but we did not find links between the microbiome and local progression of PCa. However, the correlated expression of Pseudomonas genes and human small RNA genes may provide tantalizing preliminary evidence that Pseudomonas infection may impede metastasis.

Keywords: Metagenome, Metatranscriptome, Microbial infection, Prostate cancer, Pseudouridylation

\section{Background}

Prostate cancer $(\mathrm{PCa})$ is the most common malignant neoplasm among men in Western industrialized countries and its incidence is rapidly increasing in China. Globally there are 800,000 diagnoses and 300,000 deaths annually [1]. The aetiologies of the disease remain largely unknown. Since most precancerous and cancerous tissues show signs of inflammation, chronic bacterial prostatitis has been hypothesized to be a possible etiology [2-4]. In vitro cellular

\footnotetext{
* Correspondence: renshancheng@gmail.com; ccollins@prostatecentre.com ${ }^{8}$ Department of Urology, Shanghai Changhai Hospital, Second Military Medical University, Shanghai, China

${ }^{3}$ Vancouver Prostate Centre, Vancouver, Canada

Full list of author information is available at the end of the article
}

experiments have demonstrated that inflammation induced by Escherichia coli and Propionibacterium acnes can alter normal prostate epithelial cell differentiation and through this process inflammation accelerates the initiation of PCa with a basal cell origin [5-8]. Evidence also comes from epidemiological studies as up to $87 \%$ PCa patients have microbial DNA in their prostates. In particular, $P$. acnes, JC polyomavirus (JCV) and BK polyomavirus (BKV) have been more commonly detected in $\mathrm{PCa}$ patients than in controls [9-11].

Most of the above findings were obtained by performing traditional nucleic acid amplification tests, such as quantitative real-time PCR and amplification of $16 \mathrm{~S}$ rRNA in

(c) The Author(s). 2019 Open Access This article is distributed under the terms of the Creative Commons Attribution 4.0 International License (http://creativecommons.org/licenses/by/4.0/), which permits unrestricted use, distribution, and reproduction in any medium, provided you give appropriate credit to the original author(s) and the source, provide a link to the Creative Commons license, and indicate if changes were made. The Creative Commons Public Domain Dedication waiver (http://creativecommons.org/publicdomain/zero/1.0/) applies to the data made available in this article, unless otherwise stated. 
concert with plasmid cloning and Sanger sequencing. The advent of next generation sequencing has revolutionized the study of human microbiomes. The body sites that have been intensely studied include gut, skin, oral cavity, vagina, but prostate has been largely overlooked. To date, only two studies have applied massively parallel $16 \mathrm{~S}$ rRNA sequencing for investigating the prostate microbiome [12, 13]. While these two studies confirmed that the prostate was a non-sterile environment and discovered numerous bacterial organisms that had not been reported in prostate tissue, their limited sample size $(<20$ patients) made it difficult to establish a reproducible link between microbial pathogens and PCa. Meanwhile, the innate flaws of the $16 \mathrm{~s}$ rRNA-based technologies, such as the amplification bias that may distort the bacterial composition, an inability to quantify the actual microbial load, as well as an inability to capture viruses that lack 16S rRNA, also preclude an accurate and complete characterization of prostate microbiome.

To address these limitations, we applied a shotgun-based integrated metagenomic and metatranscriptomic analysis to a cohort of 65 Chinese PCa patients. Moreover, the availability of host transcriptome offers the possibility to explore the interaction between the host cancerous tissue and its microbiome.

\section{Methods}

\section{Patient selection and specimen processing}

The information of patients and specimens is summarized in Additional file 1 [14]. Briefly, 65 PCa patients were recruited in this study, with an average age of $68.4 \pm 7.3$ years old. Treatment-naive prostate tumour and matched benign tissues were collected from the radical prostatectomy series at Shanghai Changhai Hospital and Fudan University Shanghai Cancer Center. The intact prostate glands were delivered to the pathology laboratory under sterile conditions. H\&E slides of frozen human tumour tissues and their matched benign tissues were examined by a pathologist and a urology pathologist to confirm histological diagnosis and Gleason Score. The frozen sections were then used for DNA/RNA isolation.

\section{DNA and RNA sequencing}

DNA and RNA preparation and library construction has been described [14]. In detail, for DNA library construction, DNA was extracted by phenol-chloroform and purified by the ethanol precipitation method. Then $2 \mu \mathrm{g}$ of genomic DNA from each sample was fragmented using a Covaris Ultrasonicator ${ }^{\circ}$ (Covaris, USA) to mean sizes of $500 \mathrm{bp}$. After fragmentation, the purified, randomly fragmented DNA was treated with a mix of T4 DNA polymerase, Klenow fragments, T4 polynucleotide kinase and $\mathrm{dNTPs}$ for repairing the ends by blunting and phosphorylation. The blunted DNA fragments were subsequently 3 '-adenylated using the Klenow (3'-5'exo) and ligated by
T4 DNA ligase (Rapid) (Enzymatics, USA) to PE Index Adapters. After each step, the DNA was purified using the QIAquick PCR Purification Kit (Qiagen, Germany).

For RNA library construction, RNA was extracted using TRIzol reagent and then treated with DNase I, RNase-free (Thermo Fisher Scientific, USA). Ribosomal RNA was removed from total RNA by using Ribo-Zero ${ }^{\circ}$ rRNA Removal Kit (Epicentre, USA). Then RNA was fragmented on Covaris Ultrasonicator to mean sizes of $\sim 200 \mathrm{bp}$, and the first cDNA strand was synthesized from fragmented RNA using PrimeScript $^{\circ} \mathrm{RT}$ reagent Kit (Takara Bio, Japan). After purification with the Ampure XP Beads to remove dNTPs, second-strand synthesis was performed by incubation with RNase $\mathrm{H}$, DNA polymerase, and dNTPs that contain dUTP in place of dTTP. A single 3' 'A' base was added using Klenow (3'-5' exo-) (Enzymatics, USA) and dATP to the end-repaired cDNA. Upon ligation with the PE Index Adaptors, the products were gel-recovered and subsequently digested with Uracil-N-Glycosylase (UNG) for removing the second-strand cDNA. Samples were then amplified by 15 cycles of PCR with Platinum Pfx DNA polymerase (Invitrogen, Thermo Fisher Scientific, USA).

All the constructed libraries were finally sequenced on HiSeq 2000 platform, producing $2 \times 90$-bp paired-end reads.

Filtering human DNA and mapping to microbial genomes Raw reads that contained adaptor sequences, too many Ns $(>10 \%)$ and/or low quality base ( $>50 \%$ bases with quality $<5)$ were removed. Clean reads were aligned to human reference genome hg19 using the Burrows-Wheeler Aligner (BWA-MEM v0.7.5, http://bio-bwa.sourceforge.net/). PCR or sequencing optical duplicates were marked by Picard (v1.54, http://broadinstitute.github.io/picard).

The aligned reads were removed, and the unmapped reads were then mapped by BWA to National Center for Biotechnology Information (NCBI) full set of microbial reference genomes (Bacteria: https://www.ncbi.nlm.nih.gov/ genome/microbes//; virus: https://www.ncbi.nlm.nih.gov/ genomes/GenomesGroup.cgi?taxid=10239\&host=human).

RepeatMasker (v4.0, http://www.repeatmasker.org) was used to identify repeat and low complexity reads. Any reads with three or more masked nucleotides were discarded for the next step. In order to further avoid false identification of bacterial reads which is mostly manifested as a large number of mapped reads being restricted to a few short genomic regions, the coverage uniformity of the bacterial genomes was assessed as described previously [15].

\section{Microbiome analyses}

The taxonomic abundance was measured by the number of reads assigned to each microbial genus. We hypothesize that each human cell in the specimens have 
equal amount of human DNA/RNA. Accordingly, the purpose of normalization in this study was to minimize the bias caused by differences in sequencing coverage across samples and to make the human DNA/RNA comparable between specimens. In detail, for the metagenome analysis, a scaling normalization was done by multiplying the read counts by a constant so that each specimen has $1 \times 10^{9}$ reads that were mapped to the human genome hg19. For the metatranscriptome analysis, a scaling normalization was done by multiplying the read counts by a constant so that each specimen has $1 \times$ $10^{4}$ reads that were mapped to the housekeeping gene GAPDH (ENSG00000111640). The numbers of raw and normalized reads are listed in Additional file 2.

The indices of alpha- and beta- diversity were calculated using the QIIME package (v1.9, http://qiime.org/). Comparison of these indices between groups was conducted using Student's t-test. The Non-Metric Multi-Dimensional Scaling (NMDS) analysis was performed using the vegan package in R software v3.3.

Paired comparisons of the read counts between tumour and benign specimens were conducted using Wilcoxon matched-pairs signed rank test. The $p$-values were corrected for false discovery using the Benjamini \& Hochberg $(\mathrm{BH})$ method. Only the genera with $\mathrm{BH}$-adjusted $p$-value $<0.05$ and fold-change $>2$ were considered as significantly different between specimen groups.

\section{Correlation analysis of bacterial and host expression}

Nonparametric Spearman correlation was calculated between the bacterial genes, genera and the host genes as well as the clinical parameters, including Gleason Score and prostate-specific antigen (PSA) level, in terms of their values among the 130 specimens. The returned $p$-values were corrected for false discovery using $\mathrm{BH}$ method. A Spearman correlation coefficient $>0.7$ as well as a $\mathrm{BH}$-adjusted $p$-value $<0.05$ was taken as the threshold for a significantly strong relationship.

The sequences of the bacterial genes were obtained by de novo assembly using Trinity software (v2.4, https:// github.com/trinityrnaseq/trinityrnaseq/wiki). Annotation of these transcripts was performed by BLAST search of the assembled nucleotide sequences against NCBI non-redundant protein library. The read counts of these bacterial genes were normalized with the same method as the metatranscriptome analysis.

\section{Kaplan-Meier (KM) survival analysis}

The Cleveland Clinic Foundation (CCF) cohort [16] and the JHMI cohort [17] were used for the KM analysis. The patients in the cohorts were split into two subgroups by the mean expression value of the three small RNA genes, respectively, and then the Kaplan-Meier survival analysis was performed using $\mathrm{R}$ package "survival" (http://cran.r-project.org/web/packages/survival/index. html). Weighted Cox regression models (survival 2.38-3) were used to generate KM curve $p$-values.

\section{Results}

\section{Microorganisms identified in prostate}

The cohort in this study is comprised of 65 matched PCa tumours and adjacent benign tissue from Chinese patients who underwent radical prostatectomy. Whole genome sequencing yielded 176-330 Gb between specimens, and whole transcriptome sequencing ranged from 5 to $22 \mathrm{~Gb}$. After filtering human RNA and mapping against microbial genomes, the abundance of bacterial reads derived from metagenome varied from 69 to 101,542 reads per $10^{9} \mathrm{hu}$ man reads; accordingly, the multiplicity of infection (MOI) varied from $6.8 \times 10^{-5}$ to 0.1 bacterial cells per human cell when assuming an average size of bacterial genomes to be $3 \mathrm{Mb}$. Similarly, most reads derived from metatranscriptome were also of human origin (>97\%), with the bacterial abundance ranging from $3.9 \times 10^{3}$ to $3.0 \times 10^{6}$ reads per $10^{4}$ host GAPDH reads.

A total of 47 and 116 bacterial genera were identified in the metagenome and metatranscriptome, respectively, with 43 genera being present in both (Additional file 3). The most abundant genera identified from both metagenome and metatranscriptome included Escherichia, Propionibacterium, Acinetobacter and Pseudomonas (Fig. 1), although the exact quantitative compositions varied (Fig. 1 and Additional file 3).

Four viruses were identified in the metagenome and metatranscriptome, all of which belong to dsDNA viruses. However, for majority of specimens, the viral read counts were close to zero and therefore uninformative (Additional file 4).

\section{Comparison of biodiversity indices between specimen groups}

At both metagenomic and metatranscriptomic level, the tumour and benign specimens did not differ from each other in terms of the alpha-diversity of their microbiomes (paired t test, $p>0.05$; Fig. 2a, d). The beta-diversity showed that the paired specimens from the same patients had a more similar bacterial composition to each other than between different patients (paired $\mathrm{t}$ test, $p<0.05$; Fig. $2 \mathrm{~b}, \mathrm{e}$ ); the NMDS analysis could not separate tumour and benign specimens (Fig. 2c, f).

Neither the total bacterial load nor any specific genus showed significant differential distribution between the tumour and benign specimens at the metagenomic level (Wilcoxon signed-rank test, $p>0.05$ ). The situation at the metatranscriptomic level was the same.

We further divided the patients into lower and higher grade groups based on Gleason Score: the low-grade group included 6 and $3+4$; the higher-grade group 
A

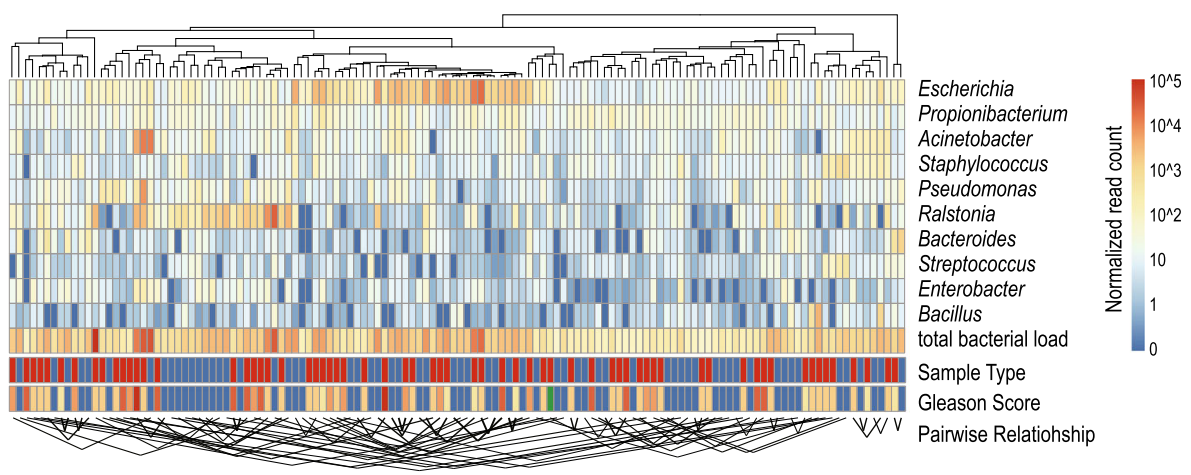

B

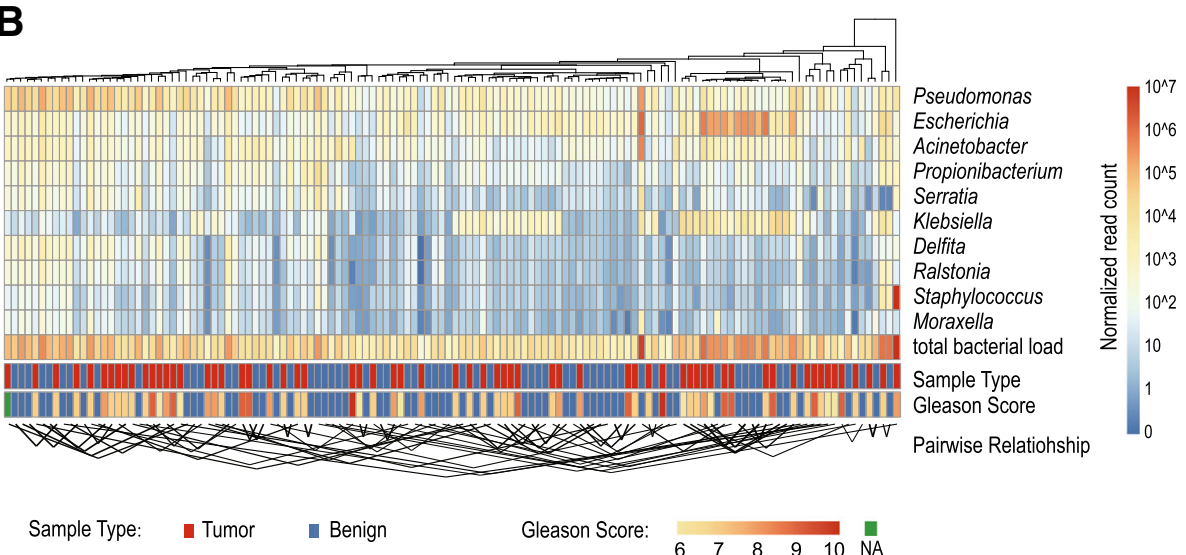

Fig. 1 Bacterial composition of prostate microbiome revealed by metagenomic (a) and metatranscripotomic sequencing (b). The upper UPGMA tree was constructed based on the weighted_UniFrac distance between specimens. The heatmap below represents the normalized read counts for the top 10 abundant genera. The lines below connect the matched tumour/benign specimens

included 8, 9, 10 and $4+3$. The alpha-diversity did not differ significantly between these two groups (paired $t$ test, $p>0.05$; Fig. $2 \mathrm{~g}$, i), and the NMDS analysis could not separate them (Fig. 2h, j).

\section{Correlation of microbiome with host expression profile}

At either metagenomic or metatranscriptomic level, no significant correlation could be found between the bacterial load (either total or of any specific genus) and any clinical parameters such as Gleason Score and prostate-specific antigen (PSA) level (Spearman correlation test, $\mathrm{r}<0.7)$. Correlations between microbial and host genes in terms of their expression profile were also investigated. Interestingly, we identified 191 pairs of host-pathogen genes that have a significantly similar expression profile (Spearman correlation test, $r>0.7$ ), nearly half of which involved ten Pseudomonas genes and eight host genes (Fig. 3). All eight host genes encode small RNA and share remarkably similar structures. Moreover, differential expression of SNORA28, RNU2-48P and SNORA40B stratified patients in terms of metastatic recurrence in another two well-studied cohorts $[16,17]$, suggesting that the infections caused by
Pseudomonas spp. and the associated expression of these small RNAs are negatively related with metastasis.

\section{Discussion}

This is the first study employing an integrated metagenomic and metatranscriptomic approach to investigate human prostate microbiome. Escherichia, Propionibacterium, Acinetobacter, and Pseudomonas were found to be abundant in both the metagenome and metatranscriptome and thus constitute the core of the prostate microbiome in this Chinese population. Escherichia and Propionibacterium have previously been shown to stimulate progression of $\mathrm{PCa}$ in in vitro experiments $[6,8,9]$, but the biological significance for the other bacteria identified in this study remains unclear. As expected, many of the organisms identified in the prostate tissue have also been detected in urine, semen, and expressed prostatic secretions [18-21]. This similar microbial composition provides evidence for the theory of ascending transmitted infection in the male urogenital tract to the seminal vesicles and prostate.

Several previous studies have associated a history of sexually transmitted infections with the risk of prostate 


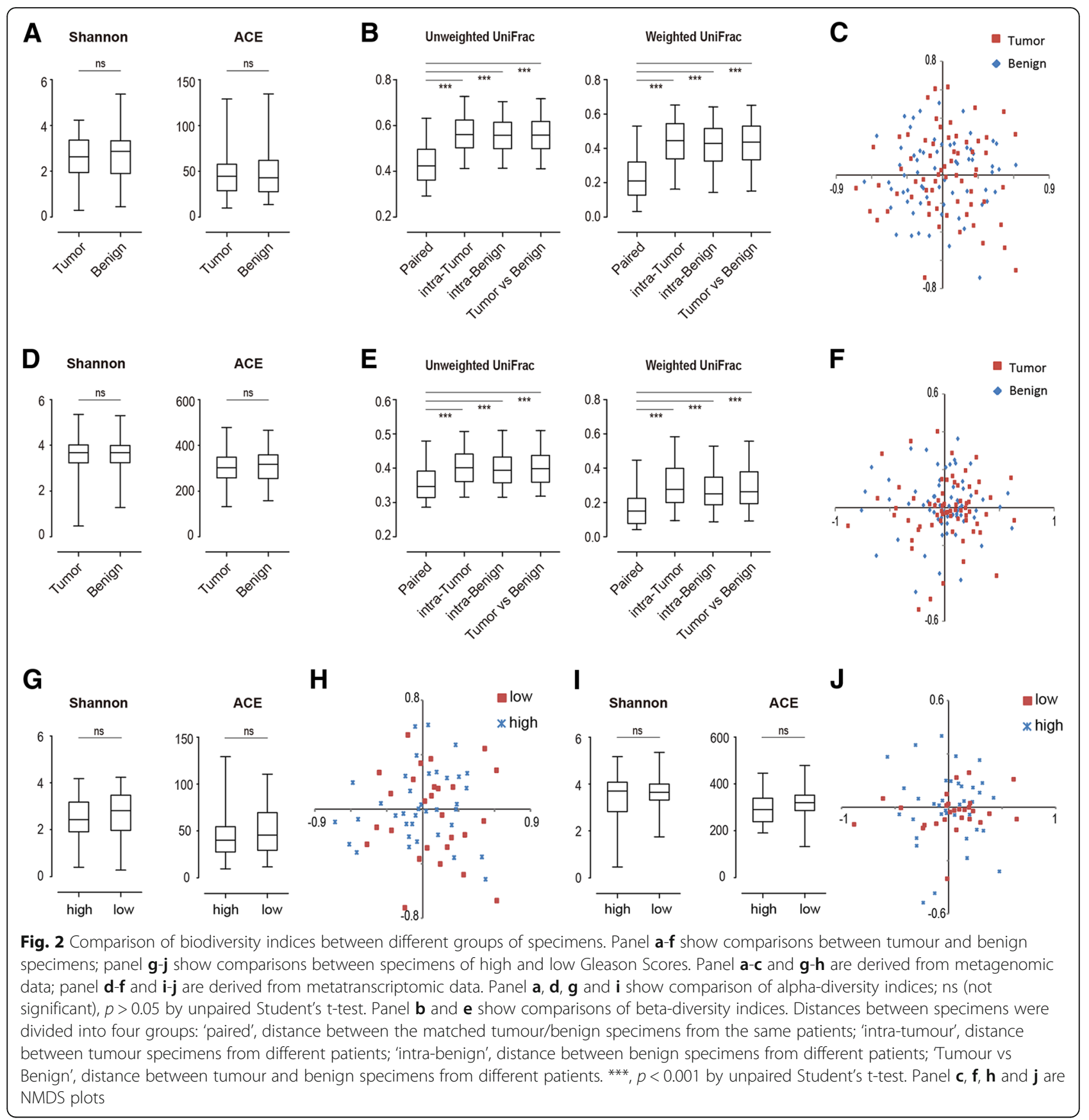

cancer [22-24]. Nevertheless, we did not detect sexually transmitted disease-related organisms in this study, such as Neisseria gonorrhoeae, Chlamydia trachomatis, Mycoplasma hominis or Ureaplasma urealyticum. Further, we did not detect viruses reported to be present in prostate tissues such as JCV, BKV, human papillomavirus (HPV) or human cytomegalovirus (HCMV) [11, 25-27]. Thus untreated sexually transmitted infections or viral infections are not expected to account for a significant proportion of $\mathrm{PCa}$ risk in the general male population, or at least in the investigated cohort.
The most urgent question in this field is whether the microbiomes from the tumour and benign tissues differ from each other and whether this difference has a causal relationship with carcinogenesis. In this study, we did not find any bacterial species that showed significantly differential distribution between tumours and their matched benign specimens at either DNA or RNA level. Also we did not find a significant difference between the tumour specimens of low and high Gleason Scores. These findings suggest that the identified microbiota may comprise the normal flora of the prostate. Due to the close proximity of the regions 

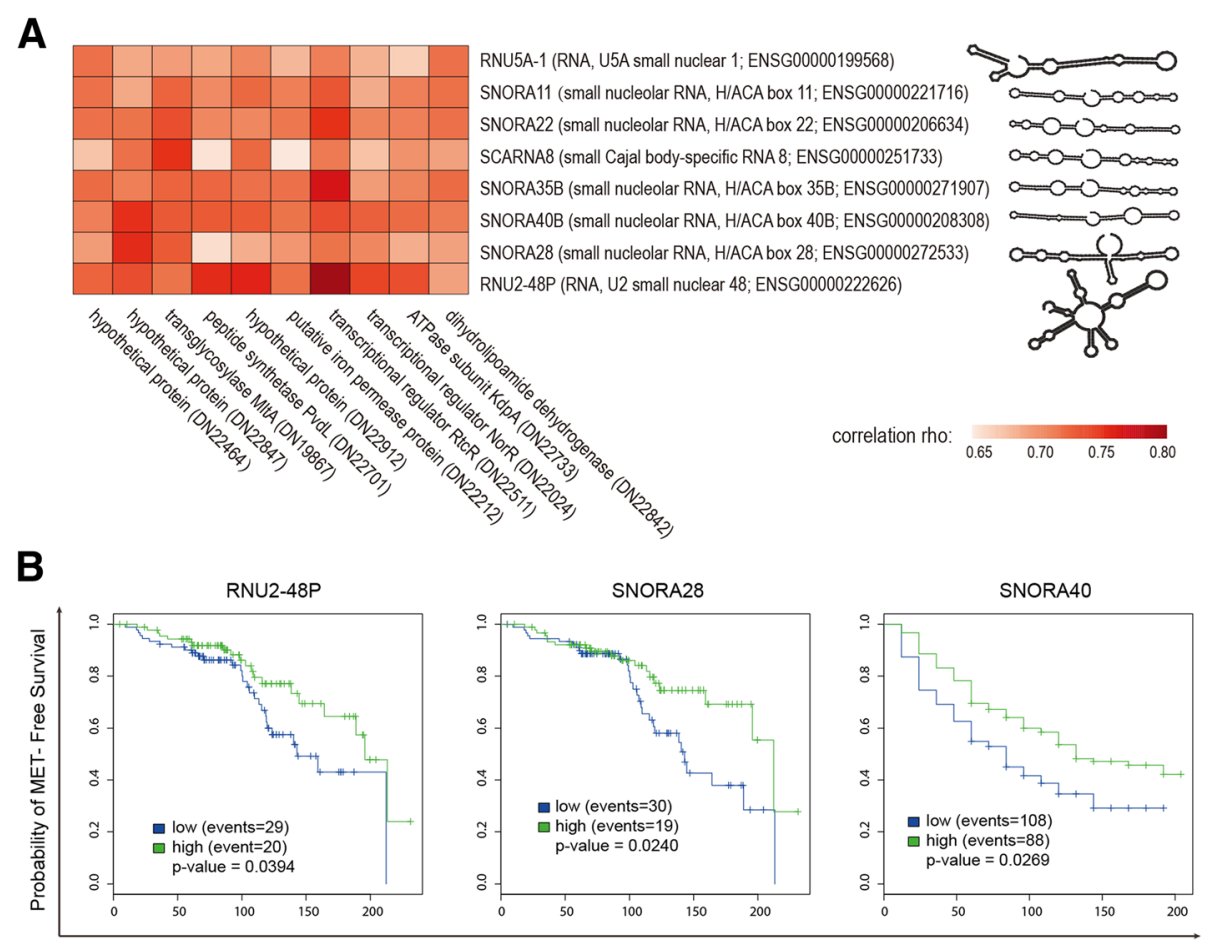

Time (Months)

Fig. 3 Correlation between bacterial metatranscriptome and host gene expression. Panel a shows the Spearman correlation values between ten Pseudomonas genes (listed below) and eight host genes (listed right). The predicted secondary structures for these host genes are displayed. The detailed annotation and nucleotide sequences of these bacterial genes are listed in Additional file 5. The Kaplan-Meier (KM) plots in panel $\mathbf{b}$ categorize the patients into the low and high group based on a median split of expression of the three small RNA genes. The higher the probability on the $y$-axis, the higher the chance of these patients NOT having metastatic recurrence (MET). The $p$-value of KM curve was generated by Weighted Cox regression models. The analysis for RNU2-48P and SNORA28 used the Cleveland Clinic Foundation (CCF) cohort [16], and the analysis for SNORA40 used the JHMI cohort [17]

compared and the field effect, we still cannot entirely exclude a causal role for bacterial infection and local PCa progression. To fully address this question, non-diseased prostate specimens are required to determine whether a healthy prostate is normally sterile or has a specific microbial flora.

The availability of the host transcriptome allowed us to detect a correlation between expression of human small RNA genes and Pseudomonas genes. Functional annotation has assigned these small RNAs to pseudouridylation, which is a major form of post-transcriptional RNA modification. Through pseudouridylation these small RNAs participate in the regulation of gene expression and therefore affect pre-mRNA splicing, translation fidelity and possibly mRNA stability and decay $[28,29]$. It has been established that pseudouridylation can be induced as a response to external stress, such as heat shock or nutrient deprivation [30]. High expression of these small RNAs in a subset of patients with low rates of metastasis suggests a negative association between Pseudomonas infection and metastasis. If this association is validated in larger cohorts then the expression profile of the bacterial and small RNA genes may be used as biomarker for active surveillance.

\section{Conclusion}

In this study we employed an integrated metagenomic and metatranscriptomic approach to investigate the prostate microbiome of PCa patients. Bacteria were detected in all specimens but the composition was not significantly different between the matched tumour and adjacent benign tissues or between different tumour grades. We also identified a strongly correlated expression profile between Pseudomonas genes and human small RNAs that may be related to metastasis. The exact mechanism of this host-pathogen interaction awaits future research.

\section{Additional files}

Additional file 1: Clinical pathological information of study cohort. (XLSX $14 \mathrm{~kb}$ )

Additional file 2: The number of raw and normalized reads for each taxonomic unit for both metagenome and metatranscriptome. (XLSX 337 kb)

Additional file 3: Comparison of bacterial composition between metagenome and metatranscriptome. (A) Venn diagram of bacterial genera identified by metagenome, metatranscriptome and the study by Yow et al. [13]. (B) The NMDS plot shows that the metagenomic and 
metatranscriptomic data could be clearly separated in terms of bacterial composition. Each dot represented a specimen. (TIF $217 \mathrm{~kb}$ )

Additional file 4: Prostatic virome. The heatmap represents the normalized read counts for the identified viruses. (TIF $236 \mathrm{~kb}$ )

Additional file 5: Detailed annotation and nucleotide sequences of the 10 bacterial genes that have the correlated expression profile with the eight small RNA genes. (XLSX $25 \mathrm{~kb}$ )

\section{Abbreviations}

BH: Benjamini \& Hochberg; BKV: BK polyomavirus; BWA: Burrows-Wheeler Aligner; CCF: Cleveland Clinic Foundation; HCMV: Human cytomegalovirus; HPV: Human papillomavirus; JCV: JC polyomavirus; KM: Kaplan-Meier; MET: Metastatic recurrence; MOI: Multiplicity of infection; NMDS: Non-Metric Multi-Dimensional Scaling; PCa: Prostate cancer

\section{Acknowledgments}

The authors thank Dr. Eric Klein, Dr. Bruce J. Trock and Dr. Yinghao Sun for providing the cohort information used in this study.

\section{Funding}

The study was funded by China Scholarship Council (CSC201606325044 to YF), Terry Fox Foundation (201012TFF to CC), Prostate Cancer Canada Team Grant (T2013-01 to CC), National Natural Science Foundation of China (81472397 to SR).

\section{Availability of data and materials}

All data supporting the conclusions of this article are included in this article and its additional files. The datasets (including whole genome and whole transcriptome sequencing data) generated and/or analysed during the current study are available in the European Genome-phenome Archive (EGAS00001000888).

\section{Authors' contributions}

YF, SR and CCC designed and initiated the study. VRR, RB, SV and ED analysed the data. YF wrote the manuscript with support of VMH. CCC coordinated the study and edited the manuscript. All authors read and approved the final manuscript.

\section{Ethics approval and consent to participate}

The study protocol was approved by the ethics committee at Shanghai Changhai Hospital. Participants gave written informed consent to participate and for investigators to obtain access to their medical records.

\section{Consent for publication}

Not applicable.

\section{Competing interests}

Dr. Ye Feng is a member of the editorial board (Associate Editor) of BMC Genomics. He as well as other authors declares no conflict of interest.

\section{Publisher's Note}

Springer Nature remains neutral with regard to jurisdictional claims in published maps and institutional affiliations.

\footnotetext{
Author details

'Sir Run Run Shaw Hospital, Zhejiang University School of Medicine, Hangzhou, China. ${ }^{2}$ Institute of Translational Medicine, Zhejiang University School of Medicine, Hangzhou, China. ${ }^{3}$ Vancouver Prostate Centre, Vancouver, Canada. ${ }^{4}$ GenomeDx Biosciences, Vancouver, Canada. ${ }^{5}$ Genomics and Epigenetics Division, Garvan Institute of Medical Research, Darlinghurst, NSW, Australia. ${ }^{6}$ School of Health Systems and Public Health, University of Pretoria, Pretoria, South Africa. ${ }^{7}$ St Vincent's Clinical School, University of New South Wales, Randwick, NSW, Australia. ${ }^{8}$ Department of Urology, Shanghai Changhai Hospital, Second Military Medical University, Shanghai, China.
}

Received: 5 October 2018 Accepted: 16 January 2019

Published online: 18 February 2019

\section{References}

1. Global Burden of Disease Cancer C, Fitzmaurice C, Dicker D, Pain A, Hamavid H, Moradi-Lakeh M, MF MI, Allen C, Hansen G, Woodbrook R, et al. The Global Burden of Cancer 2013. JAMA Oncol. 2015;1(4):505-27.

2. Coussens LM, Werb Z. Inflammation and cancer. Nature. 2002;420(6917): 860-7.

3. Lax AJ, Thomas W. How bacteria could cause cancer: one step at a time. Trends Microbiol. 2002;10(6):293-9.

4. Nelson WG, De Marzo AM, Isaacs WB. Prostate cancer. N Engl J Med. 2003; 349(4):366-81.

5. Alfano M, Canducci F, Nebuloni M, Clementi M, Montorsi F, Salonia A. The interplay of extracellular matrix and microbiome in urothelial bladder cancer. Nat Rev Urol. 2016;13(2):77-90.

6. Kwon OJ, Zhang L, Ittmann MM, Xin L. Prostatic inflammation enhances basal-to-luminal differentiation and accelerates initiation of prostate cancer with a basal cell origin. Proc Natl Acad Sci U S A. 2014;111(5):E592-600.

7. Shinohara DB, Vaghasia AM, Yu SH, Mak TN, Bruggemann H, Nelson WG, De Marzo AM, Yegnasubramanian S, Sfanos KS. A mouse model of chronic prostatic inflammation using a human prostate cancer-derived isolate of Propionibacterium acnes. Prostate. 2013;73(9):1007-15.

8. Simons BW, Durham NM, Bruno TC, Grosso JF, Schaeffer AJ, Ross AE, Hurley PJ, Berman DM, Drake CG, Thumbikat P, et al. A human prostatic bacterial isolate alters the prostatic microenvironment and accelerates prostate cancer progression. J Pathol. 2015;235(3):478-89.

9. Davidsson S, Molling P, Rider JR, Unemo M, Karlsson MG, Carlsson J, Andersson SO, Elgh F, Soderquis B, Andren O. Frequency and typing of Propionibacterium acnes in prostate tissue obtained from men with and without prostate cancer. Infect Agent Cancer. 2016;11:26.

10. Fioriti D, Videtta M, Mischitelli M, Degener AM, Russo G, Giordano A, Pietropaolo V. The human polyomavirus BK: potential role in cancer. J Cell Physiol. 2005;204(2):402-6.

11. Samanta M, Harkins L, Klemm K, Britt WJ, Cobbs CS. High prevalence of human cytomegalovirus in prostatic intraepithelial neoplasia and prostatic carcinoma. J Urol. 2003;170(3):998-1002.

12. Cavarretta I, Ferrarese R, Cazzaniga W, Saita D, Luciano R, Ceresola ER, Locatelli I, Visconti L, Lavorgna G, Briganti A, et al. The microbiome of the prostate tumor microenvironment. Eur Urol. 2017;72(4):625-31.

13. Yow MA, Tabrizi SN, Severi G, Bolton DM, Pedersen J, Australian Prostate Cancer B, Giles GG, Southey MC. Characterisation of microbial communities within aggressive prostate cancer tissues. Infect Agent Cancer. 2017;12:4.

14. Ren S, Wei G-H, Liu D, Wang L, Hou Y, Zhu S, Peng L, Zhang Q, Cheng Y, Su $\mathrm{H}$, et al. Whole-genome and Transcriptome Sequencing of Prostate Cancer Identify New Genetic Alterations Driving Disease Progression. Eur Urol. 2017; doi: https://doi.org/10.1016/j.eururo.2017.08.027.

15. Zhang C, Cleveland K, Schnoll-Sussman F, McClure B, Bigg M, Thakkar P, Schultz N, Shah MA, Betel D. Identification of low abundance microbiome in clinical samples using whole genome sequencing. Genome Biol. 2015;16:265

16. Klein EA, Yousefi K, Haddad Z, Choeurng V, Buerki C, Stephenson AJ, Li J, Kattan MW, Magi-Galluzzi C, Davicioni E. A genomic classifier improves prediction of metastatic disease within 5 years after surgery in node-negative high-risk prostate cancer patients managed by radical prostatectomy without adjuvant therapy. Eur Urol. 2015;67(4): 778-86.

17. Ross AE, Johnson MH, Yousefi K, Davicioni E, Netto GJ, Marchionni L, Fedor HL, Glavaris S, Choeurng V, Buerki C, et al. Tissue-based genomics augments post-prostatectomy risk stratification in a natural history cohort of intermediate- and high-risk men. Eur Urol. 2016;69(1):157-65.

18. Javurek AB, Spollen WG, Ali AM, Johnson SA, Lubahn DB, Bivens NJ, Bromert $\mathrm{KH}$, Ellersieck MR, Givan SA, Rosenfeld CS. Discovery of a novel seminal fluid microbiome and influence of estrogen receptor alpha genetic status. Sci Rep. 2016;6:23027.

19. Smelov V, Arroyo Muhr LS, Bzhalava D, Brown LJ, Komyakov B, Dillner J. Metagenomic sequencing of expressed prostate secretions. J Med Virol. 2014:86(12):2042-8.

20. Whiteside SA, Razvi H, Dave S, Reid G, Burton JP. The microbiome of the urinary tract--a role beyond infection. Nat Rev Urol. 2015;12(2):81-90. 
21. Yu H, Meng H, Zhou F, Ni X, Shen S, Das UN. Urinary microbiota in patients with prostate cancer and benign prostatic hyperplasia. Arch Med Sci : AMS. 2015;11(2):385-94.

22. Hayes RB, Pottern LM, Strickler H, Rabkin C, Pope V, Swanson GM,

Greenberg RS, Schoenberg JB, Liff J, Schwartz AG, et al. Sexual behaviour, STDs and risks for prostate cancer. Br J Cancer. 2000;82(3):718-25.

23. Sutcliffe S, Zenilman JM, Ghanem KG, Jadack RA, Sokoll L, Elliott DJ, Nelson WG, De Marzo AM, Cole SR, Isaacs WB, et al. Sexually transmitted infections and prostatic inflammation/cell damage as measured by serum prostate specific antigen concentration. J Urol. 2006;175(5):1937-42.

24. Taylor ML, Mainous AG 3rd, Wells BJ. Prostate cancer and sexually transmitted diseases: a meta-analysis. Fam Med. 2005;37(7):506-12.

25. Das D, Shah RB, Imperiale MJ. Detection and expression of human BK virus sequences in neoplastic prostate tissues. Oncogene. 2004;23(42):7031-46.

26. Grinstein S, Preciado MV, Gattuso P, Chabay PA, Warren WH, De Matteo E, Gould VE. Demonstration of Epstein-Barr virus in carcinomas of various sites. Cancer Res. 2002;62(17):4876-8.

27. Zambrano A, Kalantari M, Simoneau A, Jensen JL, Villarreal LP. Detection of human polyomaviruses and papillomaviruses in prostatic tissue reveals the prostate as a habitat for multiple viral infections. Prostate. 2002;53(4):263-76.

28. Karijolich J, Yi C, Yu YT. Transcriptome-wide dynamics of RNA pseudouridylation. Nat Rev Mol Cell Biol. 2015;16(10):581-5.

29. Carlile TM, Rojas-Duran MF, Zinshteyn B, Shin H, Bartoli KM, Gilbert WV. Pseudouridine profiling reveals regulated mRNA pseudouridylation in yeast and human cells. Nature. 2014;515(7525):143-6.

30. Taylor BS, Schultz N, Hieronymus H, Gopalan A, Xiao Y, Carver BS, Arora VK, Kaushik P, Cerami E, Reva B, et al. Integrative genomic profiling of human prostate cancer. Cancer Cell. 2010;18(1):11-22.

Ready to submit your research? Choose BMC and benefit from:

- fast, convenient online submission

- thorough peer review by experienced researchers in your field

- rapid publication on acceptance

- support for research data, including large and complex data types

- gold Open Access which fosters wider collaboration and increased citations

- maximum visibility for your research: over $100 \mathrm{M}$ website views per year

At $\mathrm{BMC}$, research is always in progress.

Learn more biomedcentral.com/submissions 